\title{
Spreading of liquid drops on Agar gels
}

\author{
Mehdi Banaha ${ }^{1, a}$, Adrian Daerr ${ }^{1}$, and Laurent Limat ${ }^{1}$ \\ Laboratoire Matière et Systèmes Complexes (MSC), UMR 7057 CNRS \& Université Paris Diderot, bt. \\ Condorcet CC7056, 75205 Paris cedex 13, France
}

\begin{abstract}
We study the spreading of pure water drops or water drops with surfactine (surfactant produced by bacteria Bacillus Subtilis) on gels (Agar/Water gel). We find that, surprisingly, the drops do not spread indefinitely, but remain in a state of partial wetting. Eventually the liquid diffuses into the gel on a time scale short with respect to evaporation times. The drops containing surfactant show a complex dynamics: at first the spreading velocity decreases, until the front stops and starts receding at about constant velocity. Concurrently, a second front detaches from the rim of the drop if the agar concentration is sufficiently low, and continues to move outwards.
\end{abstract}

\section{Introduction}

Although gels have come into focus of research in recent years both as a promising technological material and a physically intriguing state of matter, their wetting properties have hardly been studied. Gels are typically very soft, and consist mainly of solvent. Both aspects are different from usual materials on which wetting is studied. Soft substrates modify the spreading dynamics $[11,7,10,9]$, and gels might behave yet differently from soft rubber due to the fact that the solvent can diffuse through the polymer network, adapting the shape to constraints[1]. From a fundamental point of view, wetting a gel with its solvent can help the understanding of wetting as it simplifies the microscopic physics of van-der-Waals interactions[2]. In biology, the wetting of gel surfaces seems to play a role for the phenomenon of bacterial mass swarming[3]: before swarming across the gel, the bacillus B. Subtilis starts to produce a surfactant named surfactine. Mutants incapable of producing surfactine lose their capacity to mass migrate, except if given surfactine. Understanding in how far wetting plays a role here was our starting point for the experiments. For this reason our experiments use Agar gels and the surfactant produced by B. Subtilis, surfactine[4].

\section{Experiment}

Agar gels are made using granulated Difco Agar from BD Company. Gel concentrations are specified as mass fraction of Agar over total mass. Either demineralized water or a nutrient solution used by biologists is added as a solvent to Agar, and the mix is heated and agitated until all Agar granules have dissolved. We then pour $25 \mathrm{~m} \ell$ into Petri dishes of $8 \mathrm{~cm}$ diameter and cover these. At room temperature, we wait at least about $25 \mathrm{~min}$ for a $\sim 5 \mathrm{~mm}$ thick gel to form. We remove the cover lid 5 min before the actual experiment.

Fig. 1 show a schematic of the experimental set-up. A drop of liquid is deposited on top of the gel, which rests on a grid pattern and is filmed from above. Unless the free surface is

\footnotetext{
a e-mail: mehdi.banaha@univ-paris-diderot.fr
} 

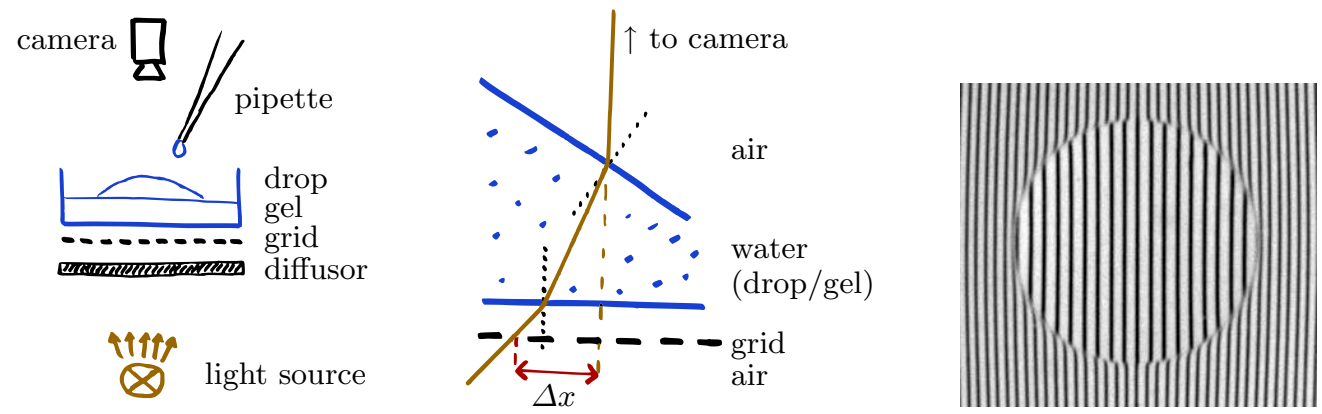

Fig. 1. Experimental set-up. Left: schematics of the lighting. Middle: more detailed side-view of the light-path through the gel and drop. When the free surface is sloped as drawn, the grid lines appear displaced by $\Delta x$ as seen from the camera, due to the refraction of the light path at the air-gel interfaces (the Plexiglas bottom of the Petri-dish is not shown in this drawing; as it is flat, it can be accounted for by a slightly increased gel thickness). Right: drop on top of the gel, as seen by the camera.
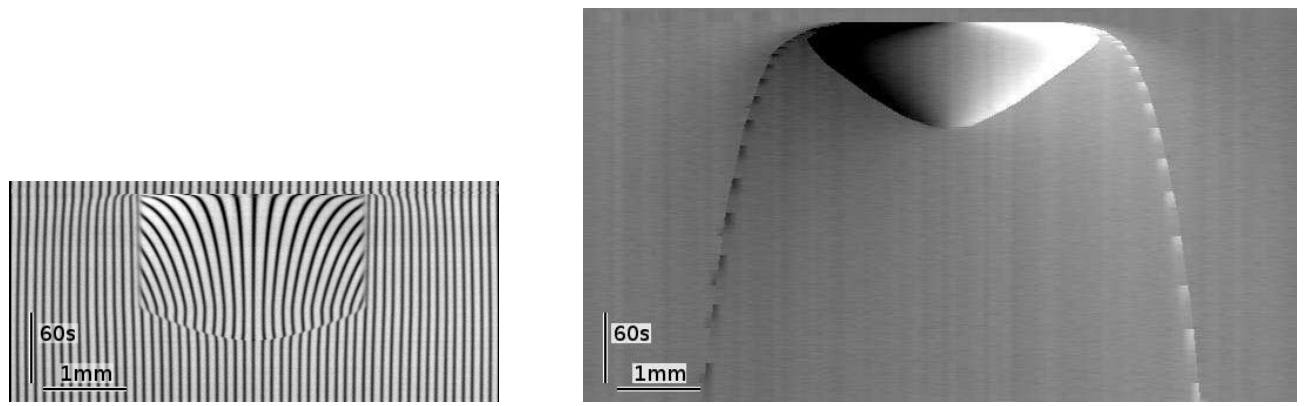

Fig. 2. Spatio-temporal representation of spreading drops. A horizontal line through the center of the drop was extracted from images as in Fig. 1 right, and plotted as a function of time (vertical axis). Time increases from top to bottom. Left: $1 \mu \ell$ water drop. Right: $1 \mu \ell$ surfactine solution $(0.25 \mathrm{~g} / \mathrm{l})$. The left view was constructed directly from a movie, showing the grid line displacements. The right view shows the local slope as calculated from the grid displacement, with positive slopes darker than grey and negative slopes lighter (i.e. as if the drop were illuminated from the right hand side).

perfectly horizontal, the image of the grid is deformed by the transparent gel/drop system, which has the optical density of water $(n \simeq 1.3)$. In our case, the deposited drop acts as a lens. Through simple geometrical optics considerations, we can relate the apparent displacement of the grid lines (with respect to an image taken before drop deposit) to the local slope of the gel/air or drop/air interface. More precisely, we obtain the local slope perpendicular to the grid lines, which is sufficient since our system generally evolves with cylindrical symmetry. We estimate the systematic relative error of the method to about $5 \%$, due essentially to the uncertainties on the required distance measurements and the small-angle approximation made on Snell-Descartes' refraction law at the interfaces. The method was checked by depositing a plane-convex glass lens of known optical index and focal length on the gel instead of the drop. The method has been optimized in order to be able to measure a large range of slopes, from $1 \mathrm{rad}$ down to about $10^{-3} \mathrm{rad}$.

We measure the slope along a line passing through the center of the drop. This slope-profile can be integrated to obtain the height profile if desired.

\section{Pure water drops}

Fig. 2 shows a spatio-temporal representation of the evolution of drops on the gel surface. It is quite surprising to see that a pure water drop on a hydro-gel (Fig. 2 left) does not spread spontaneously. The contact lines remain pinned at a non-zero contact angle (discontinuity in the 

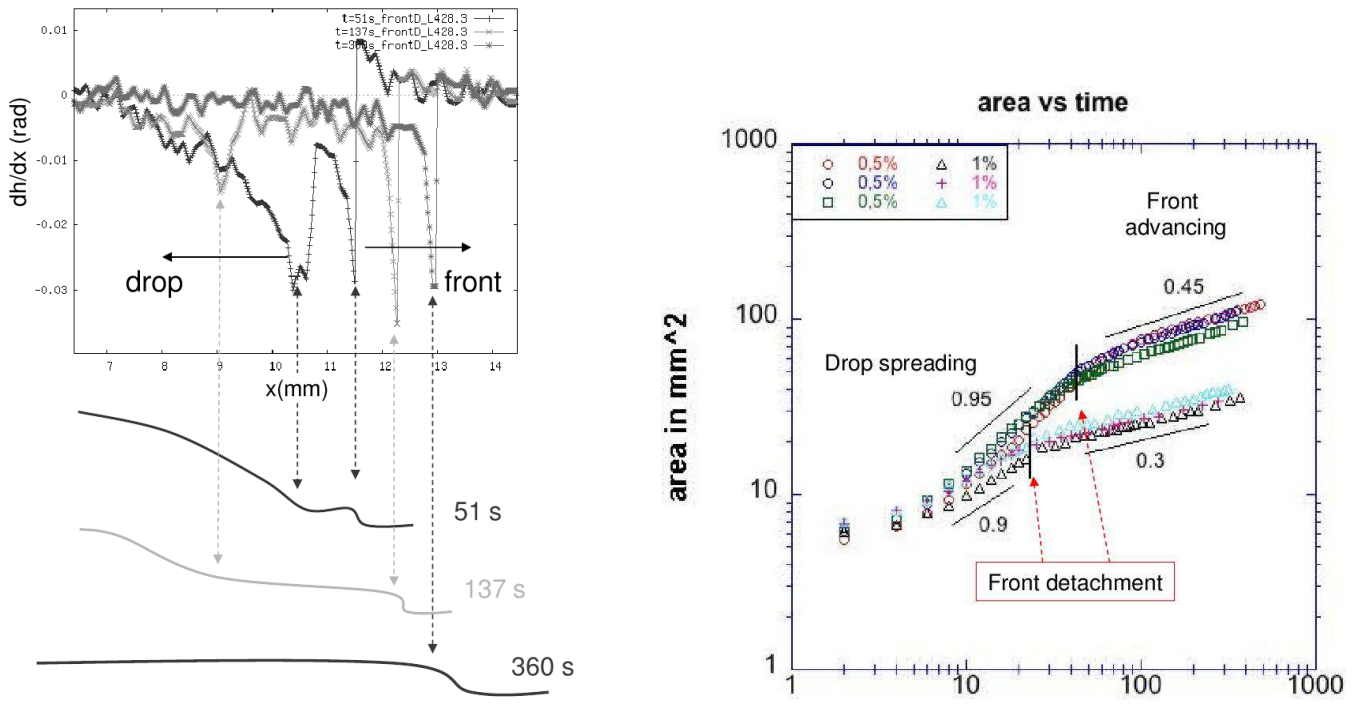

Fig. 3. Top left: slope profile from the center of the drop outwards, at three different times. The corresponding height profile is sketched below. The surfactine front moves steadily outwards, at constant amplitude, while the drop disappears into the gel. Right: The gel area enclosed by the drop rim, and later by the surfactine front, as a function of time. There is a change in spreading behavior once the surfactine front has detached, separating two roughly power-law regimes. The graph shows three series of measurements for each of two gel concentrations (0.5\% and $1 \%)$.

grid pattern), of initially around $30 \mathrm{deg}$. The drop gradually flattens (grid lines inside tighten) and after about $2 \mathrm{~min}$ starts to retract and eventually disappear. The water absorbs into the gel, which can be seen swelling around the contact line right from the moment of drop deposit. The volume change of the gel measured just after the drop has disappeared (approximatively 2 min after deposit) corresponds to about $80 \pm 10 \%$ of the initial drop volume. For comparison, we measured the time for a drop to evaporate on a hard substrate in the same set-up (relative humidity about 50 to $60 \%$ ), and found values around $30 \mathrm{~min}$, confirming that evaporation can be neglected here.

\section{Water drops with added surfactant}

Water drops with added surfactant on agar gel behave quite differently from pure water drops (Fig. 2 right). The drop still absorbs into the gel eventually, but the dynamics differs. First of all, there is no pinned state any more: the drop spreads a little bit after being deposited, and immediately crosses over to the retraction phase. Secondly, a step-like front can be seen detaching from the drop at about the moment at which the drop reaches its greatest extension. This front moves outwards, even after the drop has fully disappeared into the gel. The surface slope profile (Fig. 3 left) shows the front position (negative spikes in the slope) at different times. It is interesting to notice that the front amplitude seems to stay constant independently of time. The observation suggests that the front propagates without changing shape. This outward propagating ring-like structure is observed only relatively dilute gels $(0.5$ to $1.5 \%$ Agar). Its amplitude and velocity decrease with increasing Agar concentration, and no front is observed above about 1.5\% Agar. This concentration happens to be also, maybe accidentally, the limit above which the bacillus B. Subtilis cannot initiate swarming. Both amplitude and velocity of the front do not seem very sensitive to surfactant concentration in the relatively modest range investigated ( 0.1 to $2.0 \mathrm{~g} / \mathrm{l})$.

The spreading dynamics of the surfactant drop appears to separate into two successive stages (Fig. 3 right). At first the drop itself spreads, the contact line advancing roughly as the square root of time. After some time the drop starts retracting, but a surfactine front detaches 
and continues to move outwards. This propagation also seems to roughly follow a power law, but the exponent is much lower and possibly dependent on the Agar concentration. The only other works on drop spreading on gel that we are aware of predict/observe a $t^{1 / 2}$ power-law for the drop radius as it spreads $[8,10]$, which is compatible with our observations (exponent approx. 0.45). No front was observed in those studies however (no surfactant), so there is no direct comparison with the front propagating regime. This front dynamics is reminiscent on the other hand of spreading of surfactants on thin films of liquid, where thinning and surfactant fronts are observed $[5,6]$.

\section{Discussion}

The most surprising result is that a drop of pure water does not spread on Agar hydro-gel, even though the latter consists of up to $99.5 \%$ of water. Apparently the gel surface presents enough non-wetting defects to pin the contact line. As the drop shrinks, de-wetting occurs with a contact angle of about 2 degrees. It would be obviously interesting to know whether other hydro-gels, chemically better defined than the algae-extract Agarose, behave similarly.

Even with surfactant added to it, the drop keeps a finite contact angle and the contact line recedes as it shrinks. There is however no more noticeable pinning state. In this case, can we draw an analogy between absorption into the gel and evaporation ? It is well known that in the presence of evaporation, even wetting liquids present a finite apparent contact angle during the spreading and retracting dynamics[12]. If the absorption into the gel is a diffusive process, it is physically similar to evaporation, and the ensuing dynamics should be the same. As the contact angles do not seem do depend much on the gel concentration, the swelling itself might not affect the drop dynamics very much.

Conversely, the absorption dynamics of drops on gels could be used as a model for evaporation: it is notoriously difficult to evaporate into air in a well defined and reproducible way, especially in the diffusive regime. The swelling of a gel on the other hand is very reproducible, and easy to tune. We plan to do experiments with colloid solutions or polymer solutions to see whether the resulting deposits are similar to those seen in evaporative experiments.

The authors thank F. Lequeux, Y. Couder, S. Garoff, B. Holland and S. Seror for helpful discussions. The idea of an equivalence between absorption into the gel and drying into air came up in discussions with M. Doi and T. Yamaguchi, to whom we are indebted. This work was supported through French ACI DRAB funding, and more recently by the ANR funding DEPSEC.

\section{References}

1. T. Yamaue and M. Doi, J. Chem. Phys. 122 (2005), 084703

2. M. A. Cohen Stuart and F. A. M. Leermakers, Physica A 370 (2006) 25-257

3. D. Julkowska, M. Obuchowski, I. B. Holland and S. J. Séror, Microbiology 150 (2004) 1839-1849

4. F. Peypoux, J. M. Bonmatin and J. Wallach, Appl Microbiol Biotechnol 51 (1999) 553-563

5. A. B. Grotberg and D. P. Gaver III, J. Coll. Interf. Science 178 (1996) 377-378

6. Benjamin J. Fischer and Sandra M. Troian, Phys. Fluids 1512 (2003) 3837-3845

7. C. Extrand and Y. Kumagai, J Coll. Interf. Sc. 185 (1996)

8. D. Szabo, S. Akiyoshi, T. Matsunaga, J. P. Gong, Y. Osada and M. Zrínyi, J. Chem. Phys. 1318 (2000)

9. K. E. Daniels, S. Mukhopadhyay, P. J. Houseworth, and R. Behringer, Phys. Rev. Lett. 99 (2007), 124501

10. D. Kaneko, J. Ping Gong, M. Zrínyi and Y. Osada, J. Polymer Science B: Polymer Physics 435 (2005), 562-572

11. A. Carré and M. E. R. Shanahan C. R. Acad. Sci. Paris 317, Série II, (1993) 1153-1158

12. G. Berteloot, C.-T. Pham, A. Daerr, F. Lequeux and L. Limat, 2008 EPL 83 (2008) 14003 (6pp) 